\section{A Rare Cause of Foot Swelling Mimicking Tenosynovitis}

\section{To the Editor:}

Tenosynovitis occurring over the digital extensors of the extremities is a common cause of soft tissue swelling seen by the rheumatologist. We describe a case of long-standing soft tissue swelling on the dorsum of the foot. This was initially attributed to tenosynovitis but gradually became more edematous. Indeterminate radiological findings prompted a biopsy, which revealed an unusual low-grade tumor with metastatic potential. We highlight the importance of obtaining a soft-tissue biopsy if there is any degree of diagnostic uncertainty after radiological imaging.

A 54-year-old woman presented with a 12-month history of painful swelling over the dorsum of the right foot. The swelling varied in size, the patient describing discrete episodes of more pronounced swelling for up to 2 weeks. There was a family history of rheumatoid arthritis in the mother and sister. The patient did not experience undue weight loss or loss of appetite. Clinical examination revealed a soft, fluctuant swelling over the digital extensor tendons of the right foot (Figure 1). This measured 3-4 cm in size and was mildly tender to palpation. Rheumatoid factor and antinuclear antibody were negative. C-reactive protein and erythrocyte sedimentation rate were within normal range. The possibility of tenosynovitis was noted, with an ultrasound scan requested to confirm this.

The ultrasound showed a solid hyperemic mass with cystic elements, raising concern for the mass being neoplastic. Gadolinium enhanced magnetic resonance imaging (MRI) showed a diffuse area of abnormal signal confined to subcutaneous fat with moderate patchy enhancement. The mass did not appear to involve the extensor tendons (Figure 2). Although the appearances were fairly nonspecific, in view of the long history and persistent unilateral involvement, a referral to the regional sarcoma service was made. The patient underwent Trucut biopsies for histological examination, which confirmed an acral myxoinflammatory fibrosarcoma (AMFS). The treatment options consisted of wide local excision with reconstructive surgery or below-knee amputation. After discussion with the patient, she chose below-knee amputation to minimize the risk of local recurrence.

Soft tissue sarcomas can be difficult to distinguish from benign lesions on history and clinical examination findings alone. AMFS is part of a group of soft tissue tumors that have been recognized to mimic benign lesions ${ }^{1}$. AMFS is a low-grade tumor with a predilection for the extremities and tends to occur in middle-aged patients. It is rare, with fewer than 200 cases reported in the literature ${ }^{2}$. When situated over joints or tendons, its soft consistency may resemble synovial proliferation or tenosynovitis. Although the tumor tends to feel more firm than a benign lipoma, this may be difficult to distinguish clinically. It can also be clinically and radiographically mistaken for an atypical ganglion. AMFS is usually a painless tumor and the pain experienced by our patient may have been due to compression by footwear.

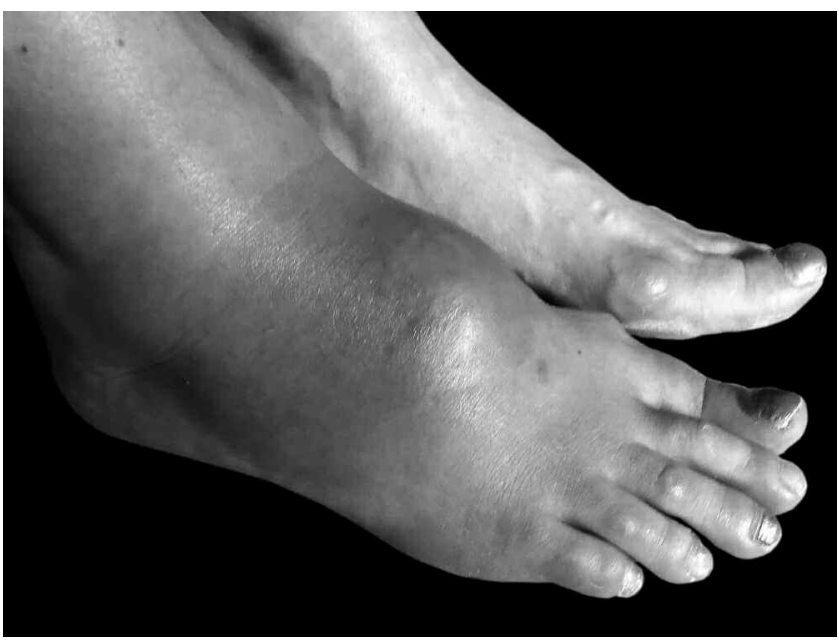

Figure 1. Soft tissue swelling in right foot.

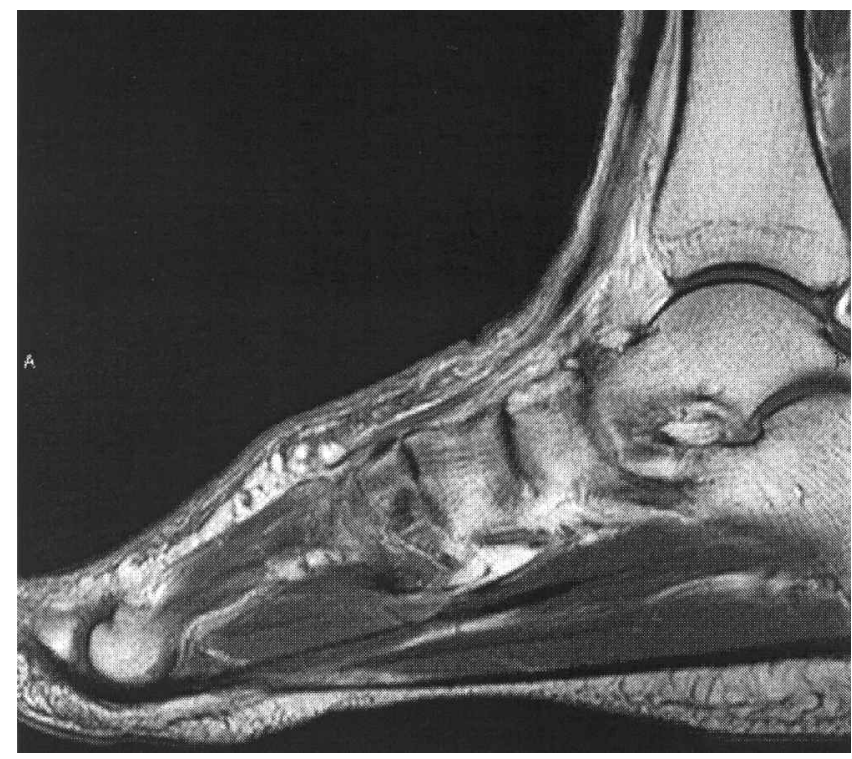

Figure 2. Gadolinium enhanced MRI showing patchy enhancement of swelling.

Radiological clarification of the swelling is important but even MRI studies have demonstrated difficulties in distinguishing this tumor from tenosynovitis $^{3}$. Histological appearances of the tumor are unusual. Large epithelioid cells with vesicular nuclei are commonly present and closely resemble the Reed-Sternberg cells seen in Hodgkin's lymphoma.

In most cases, the tumor will be confined to the dermis, underlying fascia, tendons, and ligaments (as in our patient). Muscle involvement and local bone infiltration is rare. Metastases are also rare but well described, as are tumor related deaths ${ }^{4}$. Surgical excision is the only definitive treatment, with many patients being treated with local excision only, depending on the site and extent of the tumor. Recurrence after local excision is uncommon, provided that adequate margins can be achieved.

Although many soft tissue swellings can be diagnosed clinically, our case demonstrated that a rare tumor can masquerade as a seemingly benign swelling. The protracted time course and slow-growing nature of the tumor may dissuade the clinician from a sinister pathology. However, clinical presentation of a persistent unilateral swelling, in the absence of any risk factors for foot edema, should prompt clinical suspicion of the possibility of an unusual pathology. Our case highlights the importance of confirming the nature of any soft tissue swelling with further investigations and biopsy as appropriate.

NEIL MO, BSc, MBBCh; VUN LIM, MBBCh, Rheumatology, Wrexham Maelor Hospital, Wrexham; JONATHAN J. GREGORY, BSc, MBChB; PAUL COOL, MD, MMedSci, Tumour Unit, Department of Orthopaedics, Robert Jones and Agnes Hunt Orthopaedic Hospital, Oswestry, United Kingdom. Address correspondence to Dr. Mo;

E-mail: nmo@doctors.net.uk

\section{REFERENCES}

1. Hollowood K, Fletcher CD. Soft tissue sarcomas that mimic benign lesions. Semin Diagn Pathol 1995;12:87-97.

2. Kovarik CL, Barrett T, Auerbach A, Cassarino DS. Acral myxoinflammatory fibroblastic sarcoma: case series and immunohistochemical analysis. J Cutan Pathol 2008;35:192-6.

3. Narvaez JA, Martinez S, Dodd LG, Brigman BE. Acral myxoinflammatory fibroblastic sarcomas: MRI findings in four cases. AJR Am J Roentgenol 2007;188:1302-5.

4. Hassanein AM, Atkinson SP, Al-Quran SZ, Jain SM, Reith JD Acral myxoinflammatory fibroblastic sarcomas: are they all low-grade neoplasms? J Cutan Pathol 2008;35:186-91.

J Rheumatol 2010;37:9; doi:10.3899/jrheum.100264 